\title{
Major Duodenal Papilla in Autoimmune Pancreatitis
}

\author{
Myung-Hwan Kim ${ }^{a}$ Sung-Hoon Moon ${ }^{a}$ Terumi Kamisawa ${ }^{b}$ \\ Departments of Internal Medicine, ${ }^{a}$ University of Ulsan College of Medicine, Asan Medical Center, \\ Seoul, South Korea, and ${ }^{\text {b } T o k y o ~ M e t r o p o l i t a n ~ K o m a g o m e ~ H o s p i t a l, ~ T o k y o, ~ J a p a n ~}$
}

\section{Key Words}

Autoimmune pancreatitis $\cdot$ Chronic pancreatitis $\cdot$ Duodenal papillary biopsy $\cdot \lg$ G4 immunostaining $\cdot$ Pancreatobiliary malignancies $\cdot$ Swollen papilla

\begin{abstract}
Autoimmune pancreatitis (AIP) is a distinctive form of chronic pancreatitis that can mimic pancreatobiliary malignancies. If AIP is properly diagnosed, it can be treated without laparotomy or pancreatic resection due to its dramatic response to steroid therapy. In addition to elevated serum IgG4 levels, IgG4 immunostaining of involved tissue is often used to support the diagnosis of AIP. The major duodenal papilla is a conduit between the duodenum and the pancreatobiliary system and occasionally reflects underlying pancreatobiliary disorders. Previous studies demonstrated that lgG4-positive lymphoplasmacytic infiltration was detected in the major duodenal papilla in connection to the pancreatic head in patients with AIP, and IgG4 immunostaining of biopsy specimens obtained from the major papilla could support a preoperative diagnosis of AIP. This review discusses macro- and microscopic findings of the major duodenal papilla of AIP patients, especially focusing on the diagnostic value of IgG4 immunostaining of duodenal papillary biopsy specimens.
\end{abstract}

Copyright $\odot 2010$ S. Karger AG, Basel

\section{Introduction}

Autoimmune pancreatitis (AIP) is a now widely accepted distinctive form of chronic pancreatitis that can mimic pancreatobiliary malignancies [1-3]. With no spe- cific serologic marker, diagnosing AIP is accomplished by a combination of imaging, and laboratory and pancreatic histologic findings $[2,4,5]$. As obtaining pancreatic tissue in a minimally invasive manner remains challenging, the search for a preoperative diagnosis of AIP without pancreatic biopsy has continued.

The major duodenal papilla corresponds anatomically to the junction of the common bile duct and the main pancreatic duct [6]. In addition to the function of the major papilla as a conduit between the duodenum and the pancreatobiliary system, duodenoscopic finding of the major papilla may reflect underlying pancreatobiliary disorders. For example, acute papillitis may be observed in patients with cholangitis and/or bile duct stones [6]. Secondly, the major papilla could show disease-specific findings, such as a patulous papilla exuding mucus in the case of an intraductal papillary mucinous neoplasm of the pancreas [7].

Previous studies suggested that the macroscopic appearance of the major papilla may differentiate AIP from other pancreatobiliary diseases such as malignancies [8, 9]. Additional studies revealed that microscopic findings with immunohistochemistry of the major papilla could support the diagnosis of AIP $[10,11]$. This article reviews the literature published about the major papilla in AIP and our observations.

\section{A Swollen Major Duodenal Papilla}

In 2002, Unno et al. [9] were the first to report that a swollen major duodenal papilla was a characteristic finding in patients with AIP. In the cited study [9], the authors

\section{KARGER}

Fax +4161306 1234 E-Mail karger@karger.ch www.karger.com

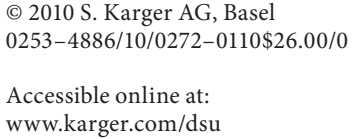

Myung-Hwan Kim, MD, PhD

Department of Internal Medicine, University of Ulsan College of Medicine

Asan Medical Center, 388-1 Pungnap-2 dong

Songpa-gu, Seoul 138-736 (South Korea)

Tel. +82 23010 3180, Fax +82 2476 0824, E-Mail mhkim@amc.seoul.kr 
classified the swelling of the major duodenal papilla by adding the scores of swollen papilla ( $0-2$ points), swollen orad protrusion ( $0-2$ points), indistinct border between the papilla and the orad protrusion $(0-1$ point), and the presence of telangiectasia ( $0-1$ point). Severe swelling of the major papilla (5-6 points) was observed in $7(41 \%)$ of 17 patients with AIP, whereas none of the patients with other pancreatobiliary diseases [11 with chronic pancreatitis, 13 with primary sclerosing cholangitis, 21 with pancreatic cancer, and 18 with bile duct cancer] had severe swelling of the major papilla [9]. Interestingly, this study [9] showed high inter- and intraobserver agreement ( $\kappa$ statistics 0.75-0.95) for a swollen papilla.

Kubota et al. [8] used an indistinct border between the papilla and the orad protrusion as their sole definition of a swollen major duodenal papilla. In their study [8], a swollen papilla was observed in $11(65 \%)$ of 17 patients with AIP and 4 (33\%) of 12 patients with chronic alcoholic tumor-forming pancreatitis. In another study by the same group using the same definition of a swollen papilla [11], a swollen papilla was recognized in $63 \%$ (17/27) of the patients with AIP compared with none of the patients with primary sclerosing cholangitis $(\mathrm{n}=12)$. The criteria for a swollen major papilla, however, may be too observer dependent to assist in the diagnosis of AIP [12].

\section{Microscopic Examination of the Major Duodenal Papilla with Immunohistochemistry}

Although other investigators suggested papillitis with immunohistochemical markers of an immune-mediated process may prompt the search for pancreatitis with an autoimmune origin $[9,13]$, Kamisawa et al. [14] were the first to report the usefulness of biopsying the major papilla to diagnose AIP, with the use of IgG4 immunostaining. According to recent studies $[10,11,15]$, when a cutoff of 10 IgG4-positive plasma cells per high-power field was applied, the sensitivity of positive IgG4 immunostaining of the major papilla was $57-80 \%$. Although the control groups were not as large, the specificity of positive IgG4 immunostaining of the papilla was extremely high (100\%) $[10,11]$.

Another group suggested that the ampulla ratio of IgG4-positive to IgG-positive plasma cells (IgG4/IgG ratio) can help differentiate AIP from other mass-forming pancreatic lesions [16]. They examined surgically resected ampulla specimens of 14 AIP, 30 pancreatic cancer and 29 chronic pancreatitis cases. The IgG4/IgG ratio seemed diagnostically useful, whereas there was some overlap in the crude number of IgG4-positive plasma cells of the papilla between AIP, pancreatic cancer, and chronic pancreatitis patients [16]. When a cutoff of 0.10 for the IgG4/ IgG ratio was applied, the diagnostic sensitivity and specificity of the ampullary $\operatorname{IgG} 4 / \operatorname{IgG}$ ratio was 86 and 95\%, respectively [16].

\section{IgG4 Immunostaining of Duodenal Papillary Biopsy Specimens: Our Experience}

To confirm the clinical validity of endoscopically accessible ampullary tissue by evaluating IgG4 immunostaining to diagnose AIP and to distinguish it from other pancreatobiliary diseases (especially malignancies), our group (Asan Medical Center, Seoul) performed a prospective research study [17].

\section{Patients and Methods}

Twenty patients with symptomatic AIP prior to steroid administration were enrolled. To allow for comparisons, 35 patients with pancreatic adenocarcinoma, 20 patients with cholangiocarcinoma, 11 patients with ampullary cancer and 18 patients with ordinary chronic pancreatitis who underwent endoscopic retrograde cholangiopancreatography (ERCP) were also included. Lastly, an additional 16 steroid-treated AIP patients who were in remission were enrolled as a comparison group. During ERCP examinations, we prospectively obtained two biopsy specimens from the major papilla under duodenoscopy (fig. 1) with pediatric biopsy forceps. Positive IgG4 immunostaining was defined as $>10$ IgG4-positive plasma cells in at least one high-power field at a magnification of $\times 400$.

\section{Results}

Comparison of IgG4 Immunostaining Positivity of the Major Duodenal Papilla between Symptomatic AIP

Patients and a Comparison Group

Positive IgG4 immunostaining of the major duodenal papilla was observed in 11 (55\%) of 20 patients with symptomatic AIP, but none of the 66 patients $(0 / 66)$ with pancreatobiliary or ampullary malignancies. Sixteen patients with AIP in remission and 18 patients with ordinary chronic pancreatitis also exhibited negative IgG4 immunostaining of the papilla. Overall, prior to steroid administration, positive IgG4 immunostaining of the major duodenal papilla resulted in $55 \%$ sensitivity and $100 \%$ specificity for the diagnosis of symptomatic AIP. 
Fig. 1. a Endoscopic image of a swollen duodenal papilla in a patient with symptomatic AIP. b Positive IgG4 immunostaining of a duodenal papillary biopsy specimen from a patient with symptomatic AIP. $\times 400$.
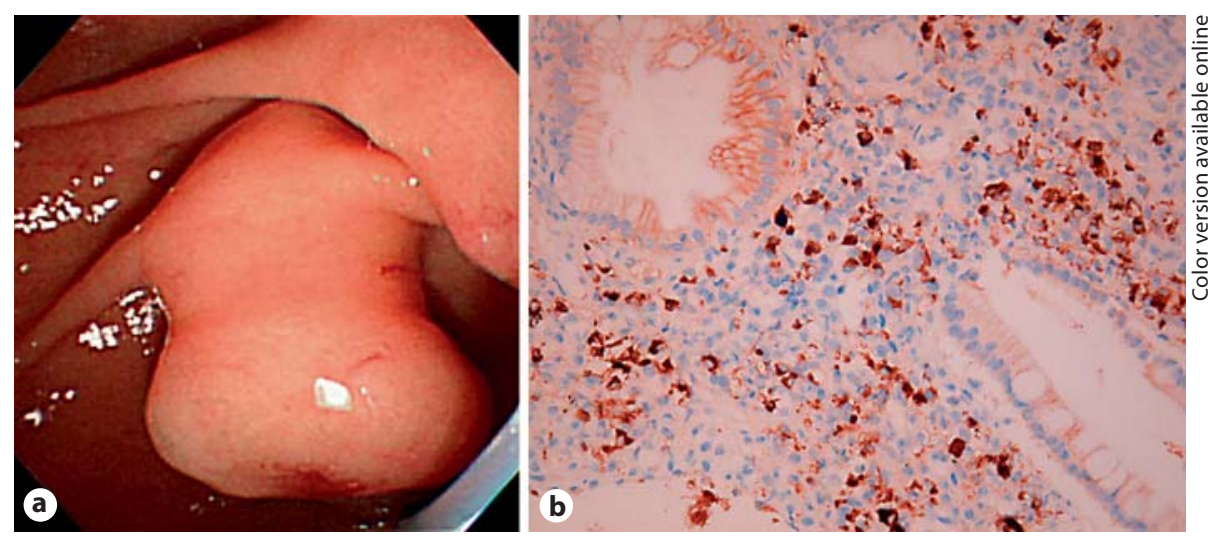

Relationship between Serum IgG4 Levels and Tissue IgG4 Immunostaining of the Major Duodenal Papilla in Symptomatic AIP Patients

In an analysis of symptomatic AIP patients, 5 (83\%) of 6 patients with elevated serum IgG4 levels showed positive IgG4 immunostaining of the major duodenal papilla, whereas $6(43 \%)$ of 14 patients with normal serum IgG4 levels showed positive IgG4 immunostaining ( $\mathrm{p}=$ $0.157)$.

Tissue IgG4 Immunostaining Positivity between the Pancreas and the Major Duodenal Papilla in Symptomatic AIP Patients

In 10 of 20 patients with symptomatic AIP, pancreatic biopsies were performed within 5 days of obtaining a papilla biopsy. Of the 6 patients with positive IgG4 immunostaining of the pancreas, 4 patients showed positive IgG4 immunostaining of the major papilla. Of the 4 patients with negative IgG4 immunostaining of the pancreas, 1 patient demonstrated positive IgG4 immunostaining of the major papilla.

\section{Relationship between the Involvement of the}

Pancreatic Head and IgG4 Immunostaining Positivity

of the Major Duodenal Papilla in Symptomatic AIP

Patients

When CT and ERCP findings were used to assess pancreatic head involvement, most patients (18/20, 90\%) with symptomatic AIP exhibited pancreatic head involvement. Positive IgG4 immunostaining of the major papilla was observed in 1 (50\%) of 2 patients with AIP who did not exhibit pancreatic head involvement compared to $10(56 \%)$ of 18 patients with pancreatic head involvement.
Relationship between a Swollen Papilla and IgG4 Immunostaining Positivity of the Major Duodenal Papilla in Symptomatic AIP Patients

A swollen papilla defined as an indistinct border between the papilla and the orad protrusion [8] was detected in 8 (50\%) of 16 patients with symptomatic AIP. Four patients who had previously undergone endoscopic sphincterotomy were excluded from the analysis of macroscopic appearance. Three of 8 patients with a swollen papilla showed positive IgG4 immunostaining of the major papilla, whereas 5 of 8 patients without a swollen papilla showed positive IgG4 immunostaining ( $\mathrm{p}=0.619)$.

There was no significant bleeding or acute pancreatitis related to endoscopic biopsy of the major duodenal papilla.

\section{Discussion}

AIP is increasingly encountered worldwide and has attracted considerable interest because of its impressive response to steroid therapy [18]. The major papilla is naturally examined in the course of a workup for the differentiation of AIP, because endoscopic ultrasonography (EUS) and/or ERCP examination is clinically needed in suspected AIP cases to rule out malignancies and/or to obtain a direct pancreatogram and/or relieve obstructive jaundice. Although previous studies $[8,9,11,15]$ showed that a swollen duodenal papilla was observed in $29-65 \%$ of the patients with AIP, its vague definition and observer-dependent nature may preclude its diagnostic use in actual practice.

Regarding IgG4 immunostaining of the major duodenal papilla, previous studies $[10,11]$ suggested this observer non-dependent method may be promising to aid 
in the diagnosis of AIP. The sensitivity of IgG4 immunostaining of the papilla, however, varies between studies (57-80\%) [10, 11, 15]. Plausible explanations for this discrepancy are as follows. First, disease activity of AIP may not be the same between these studies, and less active AIP may be more likely to exhibit negative IgG4 immunostaining of the papilla. In a previous study [10], IgG4 immunostaining of the major duodenal papilla changed from positive to negative after steroid therapy. We observed negative IgG4 immunostaining of the major duodenal papilla in all 16 AIP cases in remission, which further supports this finding. Second, the inclusion of AIP patients with idiopathic duct-centric chronic pancreatitis may influence the sensitivity of positive IgG4 immunostaining of the major duodenal papilla. Idiopathic ductcentric chronic pancreatitis often exhibits normal serum IgG4 levels and negative tissue IgG4 immunostaining in contrast to lymphoplasmacytic sclerosing pancreatitis [19]. Lastly, the number of biopsy specimens and the size of biopsy samples may affect the sensitivity of IgG4 immunostaining. In our study [17], a maximum of two papilla biopsies were obtained using small pediatric biopsy forceps to prevent possible biopsy-related pancreatitis.

Our study [17] confirmed the $100 \%$ specificity of positive IgG4 immunostaining of the major duodenal papilla in distinguishing AIP from other pancreatobiliary diseases, and included a larger number of control groups than previous studies $[10,11]$, especially with regard to pancreatobiliary malignancies, because the most important factor in diagnosing AIP is to distinguish it from pancreatobiliary cancer. Given the clinical difficulties in distinguishing AIP from pancreatic cancer $[2,3,20]$, the exceedingly high specificity of this easy and safe method may surpass its modest sensitivity.

Although elevated serum IgG4 levels are a characteristic feature of AIP, some patients with AIP exhibit normal serum IgG4 levels [21-23]. Interestingly, positive IgG4 immunostaining of pancreatic and/or extrapancreatic tissue occurred irrespective of serum IgG4 levels [21, 22]. In our patients with AIP who exhibited normal serum IgG4 levels at initial presentation $(\mathrm{n}=14)$, positive IgG4 immunostaining of the major duodenal papilla was detected in $43 \%$ (6/14). In AIP patients with normal serum IgG4 levels, therefore, the use of IgG4 immunostaining of the major papilla may be particularly useful to support a diagnosis of AIP.

Although significant infiltration of IgG4-positive cells in the 'pancreas' in patients with pancreatic cancer has been reported in the literature [24], positive IgG4 immunostaining of the 'major duodenal papilla' has not been found in patients with pancreatic cancer in our and other studies $[10,17]$. A plausible explanation is as follows: even in 'positive' (>10 IgG4-positive cells/high-power field) IgG4-immunostaining cases, the crude number of IgG4positive plasma cells is smaller in the pancreas of patients with pancreatic cancer than in those with AIP [24]. Due to a lower density of IgG4-positive cells of pancreas cancer, even if present, superficial mucosa of the major papilla which is accessible by endoscopic biopsies may not be able to reflect IgG4-positive lymphoplasmacytic infiltration of the pancreas.

A previous study [10] demonstrated that positive IgG4 immunostaining of the major papilla was observed only in AIP patients with pancreatic head involvement, suggesting that lymphoplasmacytic inflammation with increased IgG4-positive plasma cell infiltration in the major papilla may directly result from the inflamed pancreas. Our results [17], however, showed that positive IgG4 immunostaining of the major papilla was also observed in a case without pancreatic head involvement assessed by CT and ERCP. According to a recent report [25], EUS showed 'diffuse' pancreatic involvement in most cases of AIP even if CT or ERCP had only demonstrated 'focal' or 'segmental' lesions. We believe imprecision in the extent of pancreatic involvement judged by CT or ERCP may explain this discrepancy between the two studies $[10,17]$.

Chari et al. [2] recently revised their HISORt criteria. In addition to the original, other organ involvement such as hilar/intrahepatic biliary strictures, parotid/lacrimal gland involvement, mediastinal lymphadenopathy, and retroperitoneal fibrosis, the revised version of HISORt criteria added positive IgG4 immunostaining in the ampulla as a supportive diagnostic criterion. Although it is a matter of debate whether involvement of the ampulla is true other organ involvement or a direct extension of lymphoplasmacytic inflammation from the pancreatic head, IgG4 immunostaining of the major papilla can be recommended for supporting the diagnosis of AIP in a practical setting. However, the importance of pancreatic biopsy in diagnosing AIP should not be undermined as it can reliably rule out malignancy or even show histology characteristic of lymphoplasmacytic sclerosing pancreatitis.

\section{Conclusion}

A swollen papilla may be a characteristic feature of AIP, however, it may be too observer dependent to assist in the diagnosis of AIP. Positive IgG4 immunostaining of the 
major duodenal papilla may be an impeccably specific and moderately sensitive tool for the diagnosis of symptomatic AIP. IgG4 immunostaining of the papilla may offer the ability to advance a preoperative diagnosis if AIP is clinically suspected, particularly in patients with normal serum IgG4 levels or when pancreatic tissue is unavailable or nondiagnostic. In a practical setting, we recommend routine IgG4 immunostaining of duodenal papillary biopsy specimens at the time of EUS/ERCP examination in the setting of suspected AIP, as it is simple and safe.

\section{References}

${ }_{1}$ Kim KP, Kim MH, Song MH, Lee SS, Seo DW, Lee SK: Autoimmune chronic pancreatitis. Am J Gastroenterol 2004;99:16051616.

-2 Chari ST, Takahashi N, Levy MJ, Smyrk TC, Clain JE, Pearson RK, Petersen BT, Topazian MA, Vege SS: A diagnostic strategy to distinguish autoimmune pancreatitis from pancreatic cancer. Clin Gastroenterol Hepatol 2009;7:1097-1103.

3 Kamisawa T, Imai M, Yui Chen P, Tu Y, Egawa N, Tsuruta K, Okamoto A, Suzuki M, Kamata N: Strategy for differentiating autoimmune pancreatitis from pancreatic cancer. Pancreas 2008;37:e62-e67.

$\checkmark 4$ Kwon S, Kim MH, Choi EK: The diagnostic criteria for autoimmune chronic pancreatitis: it is time to make a consensus. Pancreas 2007;34:279-286.

5 Okazaki K, Kawa S, Kamisawa T, Naruse S, Tanaka S, Nishimori I, Ohara H, Ito T, Kiriyama S, Inui K, Shimosegawa T, Koizumi M, Suda K, Shiratori K, Yamaguchi K, Yamaguchi T, Sugiyama M, Otsuki M: Clinical diagnostic criteria of autoimmune pancreatitis: revised proposal. J Gastroenterol 2006;41: 626-631.

6 Park JS, Kim MH, Lee SK, Seo DW, Lee SS, Chang HS, Han J, Kim JS, Min YI: The clinical significance of papillitis of the major duodenal papilla. Gastrointest Endosc 2002;55: 877-882.

7 Pearson RK, Clain JE, Longnecker DS, Reber HA, Steinberg WM, Barkin JS, Bradley EL 3rd, DiMagno E, Layer P: Controversies in clinical pancreatology: intraductal papillary-mucinous tumor (IPMT): American Pancreatic Association Clinical Symposium. Pancreas 2002;25:217-221.

$\checkmark 8$ Kubota K, Iida H, Fujisawa T, Ogawa M, Inamori M, Saito S, Kakuta Y, Oshiro $\mathrm{H}, \mathrm{Na}-$ kajima A: Clinical significance of swollen duodenal papilla in autoimmune pancreatitis. Pancreas 2007;35:e51-e60.
-9 Unno H, Saegusa H, Fukushima M, Hamano $\mathrm{H}$ : Usefulness of endoscopic observation of the main duodenal papilla in the diagnosis of sclerosing pancreatitis. Gastrointest Endosc 2002;56:880-884.

10 Kamisawa T, Tu Y, Egawa N, Tsuruta K, Okamoto A: A new diagnostic endoscopic tool for autoimmune pancreatitis. Gastrointest Endosc 2008;68:358-361.

-11 Kubota K, Kato S, Akiyama T, Yoneda M, Fujita K, Ogawa M, Inamori M, Kobayashi N, Saito S, Kakuta Y, Ohshiro H, Nakajima A: Differentiating sclerosing cholangitis caused by autoimmune pancreatitis and primary sclerosing cholangitis according to endoscopic duodenal papillary features. Gastrointest Endosc 2008;68:1204-1208.

12 Dumonceau JM: Biliary endoscopic retrograde cholangiopancreatography. Endosco-

py 2010;42:58-61.
Sahin P, Pozsar J, Simon K, Illyes G, Laszlo F, Topa L: Autoimmune pancreatitis associated with immune-mediated inflammation of the papilla of Vater: report on two cases. Pancreas 2004;29:162-166.

14 Kamisawa T, Tu Y, Nakajima H, Egawa N, Tsuruta K, Okamoto A: Usefulness of biopsying the major duodenal papilla to diagnose autoimmune pancreatitis: a prospective study using IgG4-immunostaining. World J Gastroenterol 2006;12:2031-2033.

$\checkmark 15$ Hirano K, Fukushima N, Tada M, Isayama H, Mizuno S, Yamamoto K, Yashima Y, Yagioka H, Sasaki T, Kogure H, Nakai Y, Sasahira N, Tsujino T, Kawabe T, Fukayama M, Omata M: Diagnostic utility of biopsy specimens for autoimmune pancreatitis. J Gastroenterol 2009;44:765-773

16 Sepehr A, Mino-Kenudson M, Ogawa F, Brugge WR, Deshpande V, Lauwers GY: IgG4+ to IgG+ plasma cells ratio of ampulla can help differentiate autoimmune pancreatitis from other 'mass forming' pancreatic lesions. Am J Surg Pathol 2008;32:17701779 .

$\checkmark 17$ Moon SH, Kim MH, Park do H, Song TJ, Eum J, Lee SS, Seo DW, Lee SK: IgG4 immunostaining of duodenal papillary biopsy specimens may be useful for supporting a diagnosis of autoimmune pancreatitis. Gastrointest Endosc 2010;71:960-966.
18 Gardner TB, Levy MJ, Takahashi N, Smyrk TC, Chari ST: Misdiagnosis of autoimmune pancreatitis: a caution to clinicians. Am J Gastroenterol 2009;104:1620-1623.

19 Park DH, Kim MH, Chari ST: Recent advances in autoimmune pancreatitis. Gut 2009;58:1680-1689.

20 Moon SH, Kim MH, Park DH, Hwang CY, Park SJ, Lee SS, Seo DW, Lee SK: Is a 2-week steroid trial after initial negative investigation for malignancy useful in differentiating autoimmune pancreatitis from pancreatic cancer? A prospective outcome study. Gut 2008;57:1704-1712.

21 Deheragoda MG, Church NI, RodriguezJusto M, Munson P, Sandanayake N, Seward EW, Miller K, Novelli M, Hatfield AR, Pereira SP, Webster GJ: The use of immunoglobulin G4 immunostaining in diagnosing pancreatic and extrapancreatic involvement in autoimmune pancreatitis. Clin Gastroenterol Hepatol 2007;5:1229-1234.

22 ChariST, Smyrk TC, Levy MJ, Topazian MD, Takahashi N, Zhang L, Clain JE, Pearson RK, Petersen BT, Vege SS, Farnell MB: Diagnosis of autoimmune pancreatitis: the Mayo Clinic experience. Clin Gastroenterol Hepatol 2006;4:1010-1016.

23 Song TJ, Kim MH, Moon SH, Eum JB, Park DH, Lee SS, Seo DW, Lee SK: The combined measurement of total serum IgG and IgG4 may increase diagnostic sensitivity for autoimmune pancreatitis without sacrificing specificity, compared with IgG4 alone. Am J Gastroenterol, E-pub ahead of print.

24 Zhang L, Notohara K, Levy MJ, Chari ST, Smyrk TC: IgG4-positive plasma cell infiltration in the diagnosis of autoimmune pancreatitis. Mod Pathol 2007;20:23-28.

25 Mizuno N, Bhatia V, Hosoda W, Sawaki A, Hoki N, Hara K, Takagi T, Ko SB, Yatabe Y, Goto H, Yamao K: Histological diagnosis of autoimmune pancreatitis using EUS-guided trucut biopsy: a comparison study with EUSFNA. J Gastroenterol 2009;44:742-750. 